Europeans, the prevalence being slightly higher among boys compared to girls.

Conclusions Bissau-Guinean adolescents aged 15-16 report less smoking and drinking than European peers. Against a background of intensive marketing of alcohol and tobacco in Guinea-Bissau, it is urgent to initiate health promotional activities in secondary schools in Bissau to inform and educate adolescents and teachers on the detrimental effect of such behaviours on their long-term health and wellbeing.

\section{P46 ESTABLISHMENT OF A COMMUNITY YOUTH LEADERSHIP COUNCIL TO PROMOTE AND FACILITATE YOUTH-CENTERED HEALTH CARE DELIVERY IN A LARGE METROPOLITAN CITY}

${ }^{1} \mathrm{E}$ Uy-Smith*, 'A Labat, ${ }^{2} \mathrm{D}$ Polanco-Mendoza, ${ }^{1} \mathrm{Y}$ Teitel, ${ }^{2} \mathrm{~K}$ Morrison, ${ }^{1} \mathrm{M}$ Tapia. ${ }^{1}$ Department of Family and Community Medicine, University of California, San Francisco, San Francisco, USA; ${ }^{2}$ Community Health Programs for Youth, San Francisco Department of Public Health, San Francisco, USA

10.1136/bmjpo-2019-RCPCH-SAHM.50

Patient engagement has been touted by the Institute of Medicine as integral to effective and coordinated healthcare delivery. Scant literature exists regarding successful models of youth engagement within healthcare. In order to better address the health needs of youth in a large metropolitan city, a unique collaboration between two long-standing community health centers aims to advance youth-centered health care delivery through the establishment of a community youth leadership council (YLC). With seven decades of community engagement and health service delivery between the two clinics, the creation of the YLC is a synergy of strategic goals from both health centers. Using the expertise of the YLC, we will build upon the existing partnership between the clinics to help link this vulnerable demographic into a university-affiliated primary care medical home based on a public hospital campus. We ultimately aim to improve youth enrollment in healthcare, address health disparities in sexually transmitted infections (STIs) and healthcare utilization, and design a healthcare environment particularly welcoming to those who have been most isolated from accessing it. The YLC will assist us in establishing formal linkages with youth-facing organizations, as well as serving as consultants in creating a framework to increase pathways to primary care. We constructed a participatory process that will engage youth leaders in developing mechanisms to facilitate access for less-connected youth. Adding a community youth-operated and youth-focused cohort to the patient advisory council process will address the lack of a youth-centered perspective in clinic improvements and referral processes. Recruitment for potential YLC members was done through local high schools, clinics, and online social media. A total of 40 youth applications were received via an online- and paper-based application process. For the first three YLC meetings, at least 15 youth were in attendance at each monthly meeting. Currently planned YLC projects include standardized patient training for the youth, clinic beautification projects, and school-based 'pop-up' clinics. By bringing healthcare to the forefront of our youth's lives, the collaboration between two community health centers and the public school system will uniquely allow the youth of this large metropolitan city to transform healthcare delivery and access.

\section{P47 UNDERSTANDING THE CHALLENGES FACED BY ADOLESCENTS AND YOUNG ADULTS WITH ALLERGIC DISEASE: A SYSTEMATIC REVIEW}

${ }^{1} \mathrm{G}$ Roberts ${ }^{*},{ }^{2} \mathrm{M}$ Vazquez-Ortiz, ${ }^{3} \mathrm{~L}$ Angier, ${ }^{4} \mathrm{~N}$ Blumchen, ${ }^{5} \mathrm{P}$ Comberiati, ${ }^{2} \mathrm{~B}$ Duca, ${ }^{6} \mathrm{~A}$ DunGalvin, of EAACl Adolescent and Young Adult Task Force. 'University Child Health, University of Southampton, Southampton, UK; ${ }^{2}$ Paediatric Allergy, St Mary's Hospital, London, UK; ${ }^{3}$ Primary Care, University of Southampton, Southampton, UK; ${ }^{4}$ Pediatric Allergy, University Hospital Frantfurt, Frankfurt, Germany; ${ }^{5}$ Section of Paediatrics, University of Verona, Verona, Italy; ${ }^{6}$ Applied Psychology and Paediatrics and Child Health, University College Cor, Cork, Eire

\subsection{6/bmjpo-2019-RCPCH-SAHM.51}

Aims This systematic review aims to understand the challenges faced by adolescents and young adults (11-25 years) with allergic diseases, including asthma.

Methods A search strategy was constructed using Cochrane Database of Systematic Reviews, MEDLINE, Embase and Psychinfo. Databases were searched to February 10, 2019. Quality assessments and data extraction were undertaken independently by two reviewers.

Results 108 papers describing 106 studies were included. Most were from USA $(n=50)$, Netherlands $(n=12)$, Sweden $(n=11)$ and UK $(n=11)$. Most focused on asthma $(n=69)$ or food allergy $(n=19)$. They were qualitative, cross-sectional or longitudinal quantitative studies. There were five themes. ${ }^{1}$ Quality of life, as a measure of patients' experience of life. Factors associated with impaired quality of life: symptomatic disease, impaired psychological health, onset in adolescence, poor adherence, female sex and multiple allergies. Protective factors were exercise and increasing age. ${ }^{2}$ Psychological factors. Social anxiety has been associated with asthma and food allergy; it may inhibit patients from using medication in front of peers. Depression has also be associated with asthma and food allergy while suicidal ideation has been associated with eczema. Emotional support from family and friends has been found to be protective for asthma control. ${ }^{3}$ Adherence. Factors associated with poor adherence are older age; barriers to using medication such as lack of time, forgetfulness, cost, competing demand and accessibility, over reliance on short-acting rescue medication or poor knowledge; lack of perception of symptoms; and lack of personal responsibility. Factors associated with good adherence were routine, good self-efficacy, simple medication regimes and good knowledge about their disease. Patients who need to carry self-injectable adrenaline seem to be particularly non-adherent; the burden of these and incorrect knowledge around risks leading to poor decision making. ${ }^{4}$ Self-management. This extended from the previous theme and involved a series of barriers and corresponding facilitators. ${ }^{5}$ Supportive relationships. Key ones were identified as families, peers, friend and schools and healthcare professionals.

Conclusion The current evidence base to support the planned European guidelines for managing allergic disease in this age group is weak. Large longitudinal cohort and interventional studies are required to strength the evidence base. 